\title{
Minimally Invasive Fibrin Sealant Application in Pilonidal Sinus: A Comparative Study
}

\author{
Mahmud Saedon ${ }^{1}$, Andrew Chin ${ }^{1}$, Maryam Alfa-Wali ${ }^{2}$, \\ Chun Kheng Khoo', Aarti Varma ${ }^{1}$ \\ ${ }^{1}$ Grantham and District Hospital, Grantham, United Kingdom; \\ ${ }^{2}$ Royal London Hospital, London, United Kingdom \\ Received March 21, 2018; Accepted October 5, 2018.
}

Key words: Pilonidal disease - Fibrin glue

\begin{abstract}
The aim of this study was to compare the filling of the pilonidal sinus tract with fibrin sealant (FS) against tract excision and primary closure (PC) as the primary procedure. Details of all patients who underwent treatment for a symptomatic first episode of pilonidal sinus disease between January 2011 and December 2015 were prospectively recorded in a custom database. Patients underwent PC $(n=17)$ or FS $(n=17)$ according to patient preference. Prior surgical treatment and ongoing infection precluded entry. Patients were treated with antibiotics if presenting with infection. Outcomes measured were recurrence, further procedures, outpatient attendances and length of follow-up to resolution. 34 consecutive patients [FS vs. PC: male $n=15$ vs. $12 p=0.398$; mean age 29 (SEM 12) vs. 30 (SEM 15) $\mathrm{p}=0.849$ ] were included. Treated preoperative infections were similar FS $(n=5)$ vs. $P C(n=12)(p=0.038$, chi-squared test). FS cohort had more sinuses FS median (range) $2(1-4)$ vs. PC $1(1-3)(p=0.046)$. Postoperative outcomes: recurrence rate FS $(n=5)$ vs. $P C(n=4)(p=0.629)$; infection rate FS $(n=1)$ vs. PC $(n=8)(p=0.045)$; total number of operations required FS $1(1-2)$ vs. PC 1 $(1-4)(p=0.19)$; total number of outpatient attendance FS $2(1-7)$ vs. PC $3(1-16)$ $(p=0.629)$; follow-up FS 129 days \pm 33 vs. PC $136 \pm 51(p=0.914)$. Fibrin sealant is not inferior to excision followed by primary closure.
\end{abstract}

Mailing Address: Chun Kheng Khoo, MA, MSc, FRCS, Grantham and District Hospital, 101 Manthorpe Road, Grantham NG31 8DG, United Kingdom; Phone: +44 1476565 232; e-mail: chunkheng.khoo@nhs.net 


\section{Introduction}

Pilonidal sinus is a debilitating recurring benign condition that mainly affects young adults. Its formation arises from obstruction of hair follicles in natal cleft and subsequent rupture of follicles. The estimated prevalence is 26 over 100,000 (Sondenaa et al., 1995).

There is no consensus on optimal management. Classically, treatment is by surgical excision of sinus tract. This is followed by either primary closure or leaving wound open for healing by secondary intention; neither option has been shown to be superior. It has a significant impact on patients as the traditional techniques are associated with prolonged recovery time and delay in returning to daily life (Al-Khamis et al., 2010).

Fibrin sealant has been described as an alternative to surgical techniques (Patti et al., 2006). It consists of human fibrinogen and bovine thrombin, when combined, results in a space filling clot. The sinus is curetted, and the sealant is introduced into the cavity. The resulting reduction in dead space promotes healing, negating the need for more extensive surgical procedure and its associated complications (Kayaalp et al., 2016). Recent studies have described the use of fibrin sealant resulting with improvement in recurrent rate, early return to normal activities and high level of patient satisfaction (Lund and Leveson, 2005; Patti et al., 2006; Handmer, 2012; Elsey and Lund, 2013; Lund et al., 2017).

To date, there is no data on outcomes of comparing fibrin sealant against the conventional primary closure technique in treating pilonidal sinus disease.

The aim of this study was to compare the minimally invasive filling of the pilonidal sinus tract with fibrin sealant (FS) against tract excision with primary closure (PC), as the primary procedure for patients who presented with uncomplicated pilonidal sinus disease.

\section{Material and Methods}

The study is a retrospective analysis of a prospectively maintained database.

\section{Inclusion criteria}

All patients with symptomatic pilonidal sinus disease who underwent surgical intervention between January 2011 and December 2015. Patients with associated infection received antibiotic treatment prior to procedure. Our patients were counselled regarding the treatment options, and were given the choice of either fibrin glue or primary closure. Patients' preference determined treatment modality.

\section{Exclusion criteria}

Patients who have had previous surgery for pilonidal sinus disease, or had ongoing associated infection. 


\section{Control treatment arm}

Patients who underwent sinus tract excision followed by primary closure (PC) as the definitive and sole procedure, under general anaesthesia.

\section{Fibrin sealant treatment arm}

Patients who underwent sinus tract cleaning followed by filling of the pilonidal sinus tract with fibrin sealant (FS) as the definitive and sole procedure, under general anaesthesia.

\section{Outcomes}

Our primary outcomes were recurrences, total number of procedures required, number of outpatient attendances, and total length of follow-up until resolution.

\section{Data collection procedure}

All qualifying patients since 2011 were logged prospectively into department database. The database recorded procedures and outcomes. The data were retrieved and analysed retrospectively.

\section{Statistics}

Statistical analyses were conducted using SPSS ${ }^{\circledR}$ version 21 (Chicago, Illinois, USA).

Parametric data are expressed as mean and standard error of the mean (SEM). Non-parametric data are expressed as median and inter quartile range (IQR). Non-parametric unpaired data were analysed using the Mann-Whitney $U$ test for two group comparisons. Categorical variables were analysed using the chi-squared test or Fisher's exact test. A p-value of less than 0.05 was considered significant.

\section{Ethics}

Research Ethics Committee review was not required under the harmonised GAfREC for research limited to the use of previously collected information non-identifiable by researchers outside the usual care team. This exception also applies to research undertaken by staff within the care team using information previously collected in the course of care for the team's own patients, with the proviso that data is anonymised or pseudoanonymised in conducting the research, as such research involves no breach of the duty of confidentiality owed by care professionals. (http://www.publichealth.hscni.net/sites/default/files/directorates/files /GAfREC_changes_Remit_REC_2011_08.pdf)

\section{Results}

Baseline characteristics

In total, 34 consecutive patients were included in this study. In each treatment arm, there were 17 patients. Cohorts were similar in terms of age and sex. The mean 
Table 1 - Demographic

\begin{tabular}{lccc}
\hline & FS $(\mathrm{n}=17)$ & $\mathrm{PC}(\mathrm{n}=17)$ & P-value \\
\hline Age (mean \pm SEM) & $29 \pm 12$ & $30 \pm 15$ & 0.849 \\
Gender (male), $\mathrm{n}(\%)$ & $15(88)$ & $12(71)$ & 0.398 \\
\hline
\end{tabular}

FS - fibrin sealant; PC - primary closure; SEM - standard error of the mean

Table 2 - Preoperative clinical profiles

\begin{tabular}{lccc}
\hline & FS $(n=17)$ & $P C(n=17)$ & Chi-squared test \\
\hline Infection, $n(\%)$ & $5(29)$ & $12(71)$ & 0.038 \\
Number of sinuses (median \pm range) & $2(1-4)$ & $1(1-3)$ & 0.046 \\
\hline
\end{tabular}

FS - fibrin sealant; PC - primary closure

age was 29 (SEM 12) in the FS arm, and 30 (SEM 15) in the PC arm. Men made up $88 \%$ of patients $(n=15)$ in the FS arm, and $71 \%(n=12)$ in the PC arm (Table 1).

The PC cohort had a higher preoperative infection rate than $\mathrm{FS}$ cohort $(71 \%$ vs. $29 \%, \mathrm{p}<0.05)$. All were treated prior to surgery. The FS cohort had more number of sinuses to be treated than PC cohort (median 2 vs. 1, $\mathrm{p}<0.05$ ) (Table 2).

\section{Outcomes}

Postoperatively both cohorts had similar recurrence rate (FS 29\% vs. PC $24 \%$ ), but the postoperative infection rate was higher in PC cohort (FS 6\% vs. PC $47 \%$, $p<0.05$ ) (Table 3). All recurrent disease following fibrin sealant application were treated by lay open technique.

Table 3 - Postoperative complications

\begin{tabular}{lccc}
\hline & FS $(\mathrm{n}=17)$ & $\mathrm{PC}(\mathrm{n}=17)$ & Chi-squared test \\
\hline Recurrence, $\mathrm{n}(\%)$ & $5(29)$ & $4(24)$ & 0.629 \\
Infections, $\mathrm{n}(\%)$ & $1(6)$ & $8(47)$ & 0.045 \\
\hline
\end{tabular}

FS - fibrin sealant; PC - primary closure

\section{Table 4 - Postoperative follow-up}

\begin{tabular}{lccc}
\hline & FS $(n=17)$ & $P C(n=17)$ & Chi-squared test \\
\hline Follow-up, days (mean \pm SEM) & $129 \pm 33$ & $136 \pm 51$ & 0.914 \\
Outpatient visits (median \pm IQR) & $2(1-7)$ & $3(1-16)$ & 0.629 \\
Operations needed (median \pm IQR) & $1(1-2)$ & $1(1-4)$ & 0.190 \\
\hline
\end{tabular}

FS - fibrin sealant; PC - primary closure; SEM - standard error of the mean; IQR - inter quartile range 
There was no significant difference in follow-up requirements. The total number of procedures, the number of outpatient attendances, and the total length of follow-up were similar in both arms (Table 4).

\section{Discussion}

Fibrin sealant application in pilonidal sinus can be used in three settings; to obliterate the dead space following primary closure; to cover open wounds following lay-open technique; and as primary treatment of sinus tracts by direct filling of sinus with sealant. In our unit, we have adopted the latter as a minimally invasive approach for uncomplicated pilonidal disease.

This study suggests that the recurrence rate between primary closure and use of fibrin sealant in patients with uncomplicated sinus disease, are comparable. In addition, the use of FS neither increases the need for further procedures and outpatient attendances, nor make follow-up longer.

A recent systematic review which included 113 patients from four studies using the same sealant technique found a recurrence rate of $20 \%$, which is less than the current study (29\%) (Kayaalp et al., 2016). However, the results of this review may not be comparable. One study recruited patients with single, noncomplicated sinus tract only (Isik et al., 2014), and another was limited by a high survey non-responder rate (Elsey and Lund, 2013). All procedures in this study were performed under general anaesthesia, whereas in the review, thirty-nine percent were performed under local anaesthesia (Kayaalp et al., 2016).

We treated all recurrent disease following fibrin sealant application with a lay-open technique. Previous studies on fibrin sealant were conducted with a small number of patients and there was no information regarding the effect of repetitive application of sealant or subsequent procedures (Kayaalp et al., 2016).

A recent Cochrane review which looked into 4 RCTs (randomized controlled trials) on the use of fibrin sealant as either a monotherapy or adjunct suggested that the benefits remains unclear (Lund et al., 2017). However, the studies included had dissimilar cohorts of patients, whereas our study was specific with standardized comparative interventions. This is an observational study looking specifically into the use of monotherapy against the traditional method. Our study did not address patients with complex pilonidal sinus disease. The key aim of our study was to explore whether fibrin sealant is suitable as the first line treatment for non-complicated pilonidal sinus disease. Even though the sample size is small in the groups, the demographics in the form of age and gender are similar and this provides a good direct comparison of both treatment options.

\section{Conclusion}

There is lack of comprehensive evidence on the use of fibrin sealant. The results from this study has shown that it is not inferior to excision followed by primary 
closure. The treatment should be made available to selected groups of patients with a suitable pre-surgical profile to fully benefit from it.

\section{References}

Al-Khamis, A., McCallum, I., King, P. M., Bruce, J. (2010) Healing by primary versus secondary intention after surgical treatment for pilonidal sinus. Cochrane Database Syst. Rev. 1, CD006213.

Elsey, E., Lund, J. N. (2013) Fibrin glue in the treatment for pilonidal sinus: High patient satisfaction and rapid return to normal activities. Tech. Coloproctol. 17, 101-104.

Handmer, M. (2012) Sticking to the facts: A systematic review of fibrin glue for pilonidal disease. ANZ J. Surg. 82, 221-224.

Isik, A., Eryilmaz, R., Okan, I., Dasiran, F., Firat, D., Idiz, O., Sahin, M. (2014) The use of fibrin glue without surgery in the treatment of pilonidal sinus disease. Int. J. Clin. Exp. Med. 7, 1047-1051.

Kayaalp, C., Ertugrul, I., Tolan, K., Sumer, F. (2016) Fibrin sealant use in pilonidal sinus: Systematic review. World J. Gastrointest. Surg. 8, 266-273.

Lund, J. N., Leveson, S. H. (2005) Fibrin glue in the treatment of pilonidal sinus: Results of a pilot study. Dis. Colon Rectum 48, 1094-1096.

Lund, J., Tou, S., Doleman, B., Williams, J. P. (2017) Fibrin glue for pilonidal sinus disease. Cochrane Database Syst. Rev. 1, CD011923.

Patti, R., Angileri, M., Migliore, G., Sparancello, M., Termine, S., Crivello, F., Gioe, F. P., Di Vita, G. (2006) Use of fibrin glue in the treatment of pilonidal sinus disease: a pilot study. G. Chir. 27, 331-334.

Sondenaa, K., Andersen, E., Nesvik, I., Soreide, J. A. (1995) Patient characteristics and symptoms in chronic pilonidal sinus disease. Int. J. Colorectal Dis. 10, 39-42. 